\section{References}

JADRESIC, D. (1992) Management training - what do we need? Psychiatric Bulletin, 16, 86-87.

LitTlejohns, C. S., Wilkinson, G. \& MurPhy, E. (1992) Training psychiatrists for work in the community. Psychiatric Bulletin, 16, 23-24.
Residential Care Homes Regulations (1984). London: HMSO, 1985.

Royal College of Psychiatrists (1990) Report of the Working Group on the training implications of the move towards community oriented treatment. Psychiatric Bulletin, 14, 686-693.

Royal Commission on Medical Education 1965-1968 Report (1988). London: HMSO. Cmnd 3569.

\title{
Resuscitation skills of psychiatric trainees
}

\section{A suitable case for treatment?}

\author{
Nick Kosky, Research Fellow, West Wimbledon Community Mental Health Team, \\ Nelson Hospital, London SW20 8DB; and KEN SPEARPOINT, District Resuscitation \\ Training Officer, Dorcas House, St George's Hospital, Tooting, London SW17 0QT
}

One of the most unpleasant things that can happen to the senior house officer or registrar on call alone in a psychiatric hospital is a "crash" call. He or she has to get to the patient, institute appropriate immediate management, arrange for disposal to a more suitable facility, and hand over to the responsible team. The problems facing the psychiatrist in this situation may be complicated by several factors, and these come under the following headings.

\section{Difficulty in getting to the patient}

Psychiatric hospitals are often large institutions with an illogical layout and illogical naming system. The on-call doctor may be called to a ward he or she has not been to before, especially if the incident occurs at the beginning of their appointment. (Lack of knowledge of local layout is not just the province of psychiatric staff - Sullivan \& Guyatt, 1986).

\section{Dificulty in instituting appropriate management}

Recent studies of resuscitation skills of various groups of staff in general hospitals have shown some inadequacies (Seraj \& Naguib, 1990; Lum \& Galletly, 1989). Even cardiologists and anaesthetists were "unable to perform practical steps in the correct order" (Seraj \& Naguib, 1990). While these study populations are not directly comparable with our population, there is no reason to suppose that trainee psychiatrists, perhaps some years from their last general medical experience, would show a higher level of competence.

In addition to the doctor's possibly inadequate skills, there are additional problems in resuscitating the patient: appropriately trained nursing staff may be lacking, defibrillator machines and monitors may be slow to arrive and equipment on the "crash" trolley may be inoperative or not the same as the equipment the doctor trained on. These last problems, however, are out of the scope of this paper, as is the issue of communicating with the on call doctor about decisions regarding whether or not to resuscitate a particular patient.

The aim of this paper is to look at the theoretical knowledge and practical skills, and the knowledge of local procedures and equipment of trainee psychiatrists at one hospital.

\section{The study}

The study was divided into two parts, the first consisting of a questionnaire and the second of practical resuscitation abilities. 
The questionnaire was divided into three sections. The first section looked at the self-rated level of confidence concerning ability to deal with a cardiorespiratory arrest and what training in resuscitation and what non-psychiatric medical experience the responder had had. The second section asked about knowledge of local arrest procedures and the third, practical and theoretical aspects of resuscitation.

The practical session utilised a Laerdal Skillmeter Resusci Annie mannequin to test the ability of the trainees to make a brief assessment of a collapsed person and to maintain adequate ventilation and output for three minutes. Those taking part were then asked to identify four simple arrhythmias - atrial fibrillation, fine and coarse ventricular fibrillation, and sinus tachycardia. Lastly, they were asked to manage simulated ventricular fibrillation, using the defibrillator that would actually be taken to crash calls in this hospital, and judged according to the Resuscitation Council (UK) protocol.

We attempted to contact all the senior house officers and registrars at one large psychiatric hospital in London. Out of a possible 14, 11 were contactable. Ten agreed to fill in the questionnaire, the 11 th declining on the grounds of being too busy. The ten doctors who completed questionnaires were asked if they would attend a brief practical session; eight agreed, but only three turned up. The study was carried out at the end of an attachment period, in order that those taking part would have had the maximum opportunity to become familiar with the local arrest procedure.

\section{Findings}

Questionnaire results

Section (i) - confidence levels and resuscitation experience

Most of the ten rated themselves as "very" or "quite" confident at overall management of the first ten minutes of an arrest, maintaining organ perfusion with cardiac massage, using a defibrillator and obtaining venous access; most rated themselves as "unsure" at intubation.

Six of the trainees had received instruction in mannequin cardiopulmonary resuscitation, two had attended formal basic cardiac life support courses and two had no training in resuscitation. Of those who had training, only one had attended a course in the last year, two a year ago and the rest varying amounts up to ten years previously. Only two trainees had no post registration non-psychiatric experience; most had had between six and 18 months.

\section{Section (ii) - knowledge of local procedures}

Of the ten, five knew how to send out a crash call. Seven knew how the defibrillator was brought to an arrest, but only one knew where it was kept. None knew whether the defibrillator was semi-automatic or manual. Four had incomplete knowledge of where emergency drug supplies were kept; six had no idea. Three knew how oxygen would be brought to the arrest.

Section (iii) - knowledge of practical and theoretical aspects of resuscitation

The questions in this section were those used to assess nursing staff who have been on defibrillator training courses. The pass mark, $80 \%$, should be met by all doctors and nurses responsible for the management and delivery of care during cardiac arrests. The average mark of these trainees was $45.7 \%$, with a range of $31.5 \%$ to $52.6 \%$. The trainees did best on questions related to theoretical issues and electrophysiology and worst on those concerning practical aspects of resuscitation.

\section{Practical session results}

Only a small proportion of the junior doctors turned up and therefore results of this session should be viewed with caution; however, we believe that there is no reason for the skills of those who did not attend to be any better - the questionnaire scores and reported confidence levels of attenders and non-attenders did not differ.

Of the three doctors who did attend, none made an appropriate initial assessment of the patient; i.e. assessed their level of consciousness. Two were able to perform initial airway management, but none managed to keep the airway open for longer than this initial period and none performed adequate ventilation. None performed adequate cardiac massage. For the purpose of this study, adequate was defined as more than $80 \%$ of compressions and ventilations being performed correctly, as measured by the mannequin's inbuilt monitoring equipment. In all three cases the ratio of ventilations:compressions was incorrect.

All three doctors identified coarse ventricular fibrillation. One identified atrial fibrillation and one fine ventricular fibrillation; none could identify sinus tachycardia.

None of the three were competent at using the defibrillator and only one of them had seen it before. None of them were capable of following the protocol for the management of ventricular fibrillation beyond the first three shocks, the timing and amount of energy of which were prompted by the defibrillator.

\section{Comments}

We are aware of the small size of study population, and consequent hazards in extrapolating these results to other populations. This study is only a "snapshot" 
of the situation in one hospital; however, we feel the situation is a worrying one, and, in our experience, typical of psychiatric hospitals.

The perception of the doctors of their abilities to manage an arrest were, in the main, exaggerated. Knowledge of local crash call procedures were inadequate; although it is the nursing staff who usually put out a crash call and take equipment to an arrest, we felt it important that all those obliged to attend an arrest have an understanding of local procedure and equipment storage - especially important if, for instance, the senior nurse on duty is unable to bring any equipment.

Theoretical and practical knowledge and skills were also shown to be insufficient, especially when compared to training recommendations in the literature (The Royal College of Physicians, 1987).

Perhaps the most alarming from this study was the lack of recent, appropriate training for these doctors. It has been suggested in the literature that refresher training updates should occur at six monthly intervals (Goodwin, 1992); most of the trainees received too little training, too long ago.

One finding that emerged consistently from discussion with these doctors during this work was that none of them were clear what level of competence they should meet. Although arrest protocols for general hospitals have been published, there are no such guidelines for psychiatric hospitals. It may be that our measuring these doctors' abilities against the Royal College of Physicians' guidelines is inappropriate, but until exactly what the expectations of the on-call doctor in an arrest in a psychiatric hospital have been defined, this will remain unclear. It is a matter for debate whether, with the change in psychiatric care from institution-based to community-based and other difficulties peculiar to psychiatric hospitals, trainee psychiatrists should be expected to provide emergency medical cover; however, until this change is complete, the on-site junior doctor will be the nearest medical help to the patient, and we feel it reasonable that an arrested patient should have a fighting chance of survival until the arrival of an ambulance. This means the institution of arrest procedure guidelines, training and audit to ensure training goals are being met. A start might be to ensure that every on call doctor is capable of single- and two-person cardiopulmonary resuscitation. Until then, the expectation that psychiatric trainees continue to provide an on-site resuscitation service is an unreal one.

\section{References}

Goodwin, A. P. L. (1992) Cardiopulmonary resuscitation training revisited. Journal of the Royal Society of Medicine, 85, 452-453.

LUM, M. E. \& GALLETLY, D. C. (1989) Resuscitation skills of Ist year postgraduate doctors. New Zealand Medical Journal. 102, 406-408.

SERA, M. A. \& NAGUIB, M. (1990)Cardiopulmonary resuscitation skills of medical professionals. Resuscitation, 20, 31-39.

Sullivan, M. J. \& GuYatT, G. H. (1986) Simulated cardiac arrest for monitoring quality of in-hospital resuscitation. Lancet, 2, 618-620.

The Royal College of Physicians (1987) Resuscitation from cardiopulmonary arrest; training and organisation. Journal of the Royal College of Physicians, London, 21, 1-8.

\section{Computer workshop}

The Computers in Psychiatry Special Interest Group of the Royal College of Psychiatrists and the Senior Registrars Forum (An Educational Service for Psychiatry Wholly Sponsored by Lundbeck) are holding a two day residential workshop titled $A$ Novices Guide to the PC. This will be the first in a series of practical computer workshops for senior registrars and post Membership registrars in psychiatry and is aimed at those who consider themselves almost computer illiterate. It will be held at the College of Continuing Education, University of Oxford, Rewley House Oxford from 10 to 11 September 1993. Further information: Dr Jonathon Bisson, Sunningdale House, Caldecotte Lake Business Park, Caldecotte, Milton Keynes, MK 78 LF. 